\title{
Formación inicial de docentes de ciencia: posibles aportes y tensiones de la modelización
}

\section{Pre-service science teacher education: possible contributions and tensions on modeling}

\author{
Anna Garrido Espeja \\ Universidad Autónoma de Barcelona, Facultad de Educación, Barcelona, España \\ agarridoespeja@gmail.com \\ Macarena Soto Alvarado \\ Pontificia Universidad Católica de Chile, Facultad de Educación, Santiago, Chile \\ macarena.soto@uc.cl \\ Digna Couso Lagarón \\ Universidad Autónoma de Barcelona, Facultad de Educación, Barcelona, España \\ digna.couso@uab.cat
}

RESUMEN • El objetivo de esta investigación es conocer las percepciones de futuros docentes sobre la modelización como enfoque didáctico para la enseñanza de las ciencias identificando las tensiones didácticas que emergen entre sus preferencias metodológicas y la formación modelizadora vivida. Utilizando un cuestionario semiabierto analizamos la opinión de futuros docentes de dos contextos educativos distintos, 70 futuros docentes de primaria (de Cataluña) y 22 de secundaria (de Chile), sobre la formación vivida. Los resultados muestran que ambos grupos valoran la modelización como útil para aprender ciencias, aunque también valoran otros aspectos no propios de la modelización, con ciertas diferencias entre los dos grupos. Por último, identificamos cuatro tensiones didácticas que considerar en la planificación de cursos de formación inicial de docentes de ciencias.

PALABRAS CLAVE: Formación inicial docente; Modelización; Percepciones; Prácticas científicas.

ABSTRACT - The aim of this research is to find out the perceptions of future teachers about modeling as a teaching methodology in two different educational contexts, after participating as students in a training course based on it. We also aim to identify didactical tensions that emerge from their visions about modeling-based teaching. Using a semi-open questionnaire, we analyzed the opinion of $70 \mathrm{fu}-$ ture primary school teachers (in Catalonia) and 22 future secondary school teachers (in Chile) about their own experience. Results show that both groups value modeling as a useful practice for learning science, although they also highlight other aspects that are not specific for modeling with certain differences between the two groups, which are discussed. Finally, we identify four tensions regarding the teaching of science to consider when planning initial teacher education courses within the modeling-based instruction framework.

KEYWORDS: Pre-service teacher education; Modeling; Perceptions; Scientific Practices.

Recepción: abril 2020 • Aceptación: abril 2021

Garrido Espeja, A., Soto Alvarado, M., y Couso Lagarón, D. (2022). Formación inicial de docentes de ciencia: posibles aportes y tensiones de la modelización. Enseñanza de las Cien- 


\section{INTRODUCCIÓN}

La perspectiva que enfatiza ver el aprendizaje de las ciencias como la participación de los estudiantes en prácticas análogas a las de la ciencia es un marco que ha ido adquiriendo importancia en la investigación en didáctica de las ciencias de los últimos años (Duschl y Grandy, 2008; Osborne, 2014).

Desde la epistemología semanticista y el modelo cognitivo de ciencia, se desprende una forma particular de concebir las prácticas científicas en el aula como una actividad científica escolar (ACE), centrada en la construcción de modelos científicos escolares (MCE). Estos MCE son modelos científicos con un alto potencial descriptivo, predictivo y explicativo que han sido didácticamente reconstruidos para poder ser modelizados en el aula (Izquierdo, Sanmartí y Espinet, 1999; Izquierdo, 2005; Izquierdo, 2007). Desde esta perspectiva, con la que nos alineamos en esta investigación, la práctica científica que guía la actividad científica en el aula es la modelización, entendida como la construcción en aproximaciones progresivas de versiones cada vez más sofisticadas de estos MCE objeto de aprendizaje (Couso, 2015).

La modelización se ha reportado como relevante para la construcción del conocimiento científico, en particular los modelos científicos (escolares) (Oliva, 2019), y mejorar las visiones sobre la naturaleza de las ciencias y los resultados del alumnado con más dificultades, entre otros (Halloun, 2016). Pese a estos beneficios, las evidencias muestran que su uso en contextos escolares es escaso (Acher, Arcà y Sanmartí, 2007; Windschitl, Thompson y Braaten, 2008). Una de las principales razones es presentar el conocimiento científico como indiscutible y estandarizado (Jiménez-Aleixandre, 2010), en lugar de promover una actividad modelizadora para construirlo (Lehrer y Schauble, 2012). Además, se considera que el alumnado en edades tempranas no tiene suficientes capacidades cognitivas para el pensamiento abstracto (Acher et al., 2007). Por último, cuando los docentes intentan incorporar en el aula metodologías centradas en la modelización, aparecen muchos retos y barreras concretas (Schwarz y Gwekwerere, 2007), relacionados con unas visiones de los modelos y la modelización no siempre adecuadas (Jiménez-Tenorio, Aragón, Blanco y Oliva, 2016) y con una falta de posicionamiento crítico que cuestione el currículo o los libros de texto.

Se han realizado diferentes investigaciones en relación con formación inicial de docentes en torno a las prácticas científicas y la modelización. Schwarz et al. (2009) se centran en mejorar los conocimientos de los futuros docentes (desde ahora FD) sobre la naturaleza de la modelización y proporcionarles herramientas que les sean útiles en el aula. Otras investigaciones se han centrado en que los FD de ciencias vivan en primera persona el enfoque didáctico que desean que utilicen en el aula (como la indagación) y reflexionen explícitamente sobre él (por ejemplo, Martínez-Chico, Jiménez-Liso y LópezGay, 2014), incluyendo el papel de las emociones en este proceso (Jiménez-Liso, Martínez-Chico, Avraamidou y Lucio-Villegas, 2019).

Los antecedentes presentados justifican la necesidad de una formación inicial que dote a los FD de vivencias y herramientas que les sirven para replantearse qué metodologías usar en el aula de ciencias, promoviendo la valoración de la modelización desde la perspectiva de la ACE como una propuesta útil que incorporar en sus futuras clases. Para ello, investigamos cómo los FD de dos contextos distintos (de primaria en Cataluña y de física de secundaria en Chile) valoran la metodología de enseñanza de las ciencias centrada en la modelización en la que han participado. Además, nos centramos en identificar tensiones didácticas relevantes que surgen de la vivencia formativa de los FD, con el objetivo final de aportar ideas sobre qué características debería presentar una formación inicial de docentes en ciencias centrada en la modelización y cómo superar en el aula ciertas tensiones asociadas a la modelización. 


\section{MARCO TEÓRICO: MODELOS Y MODELIZACIÓN EN CIENCIAS}

En la literatura de didáctica de las ciencias coexisten diversas visiones de modelos científicos (Gilbert y Justi, 2016), entendidos como representaciones de objetos, procesos o fenómenos con el objetivo de describir, predecir y/o explicar (Oh y Oh, 2011). Desde la mirada semanticista que compartimos (Adúriz-Bravo, 2012), un modelo científico sirve de intermediario entre la teoría que queremos que el alumnado aprenda y el mundo de los fenómenos que queremos que sea capaz de modelizar. La ACE, como posición teórico-metodológica, consiste en involucrar a los estudiantes en las formas de hablar, pensar y hacer de la ciencia escolar, análogas a las de la ciencia real (Izquierdo, 2007), con el objetivo de hacer participar a los estudiantes en procesos de modelización para que construyan sus modelos. Desde una perspectiva curricular, los modelos objeto de enseńanza y aprendizaje de la ACE son los denominados MCE, los cuales han sido didácticamente reconstruidos y son claves para la comprensión científica del mundo (Adúriz-Bravo, 2012; Izquierdo et al., 1999).

El aprendizaje de estas pocas ideas o modelos clave (a diferencia del aprendizaje de un gran número de conceptos científicos poco importantes y compartimentados) simplifica la realidad y permite interpretar fenómenos distintos desde una misma «manera de mirar» o «reglas del juego» (Izquierdo, 2005) que son propias de cada disciplina. Aun así, la construcción de estos MCE por parte de los estudiantes no es tarea fácil. Estos vienen al aula con sus propios modelos intuitivos acerca de los fenómenos, que pueden estar más o menos de acuerdo con los MCE que deben aprender. La ACE centrada en la modelización es justamente un enfoque didáctico que permite al alumnado ir mejorando sus modelos, desde formas menos elaboradas hasta formas más sofisticadas (Couso, 2015), por lo que se acercan progresivamente al MCE objeto de aprendizaje (Couso, 2020).

Diversas investigaciones realizadas por autores de la perspectiva ACE han mostrado cómo la enseñanza por modelización es eficaz para el aprendizaje de estos MCE clave. Por ejemplo, el de energía (Soto, Couso, López y Hernández, 2017), el de ser vivo (Tena, Garrido, y López, 2018) o el de cambio geológico (Márquez y Artés, 2016).

Al igual que la idea de modelo, la modelización también es un concepto polisémico (Oliva, 2019), que hace referencia tanto a una práctica científica que aprender (un nuevo contenido o competencia) como a una manera de aprenderla (una aproximación didáctica que centra el aprendizaje de la ciencia escolar en la construcción progresiva de modelos). En este sentido, existe cierta confusión entre la modelización como práctica científica (Osborne, 2014) y como enfoque didáctico (Gilbert y Justi, 2016).

Estamos de acuerdo con Schwarz y colegas en que la modelización en el aula debería ser entendida como la participación social y personal en «dar sentido y desarrollar las propias ideas» (Schwarz et al., 2009, p. 637), más que en usar y compartir ideas científicas ya finalizadas. Pero, a diferencia de otras perspectivas sobre la modelización, desde la ACE consideramos que al modelizar el alumnado debe llegar a construir un conocimiento esencial en ciencias: los MCE (Izquierdo et al., 1999). Otros enfoques, al centrarse en una forma de hacer en el aula sin plantearse para aprender qué, a menudo limitan los contenidos que enseñar a aquellos tradicionales o que «mejor cuadran» con la metodología escogida, por lo que se reducen las demandas cognitivo-discursivas de los estudiantes y se ofrece una imagen pobre y limitada de la ciencia (Windschitl et al., 2008). El enfoque de la modelización como ACE, focalizado en construir los MCE, se centra en el proceso de construcción de un conocimiento, por definición, bien escogido para el aula. Así, aunque coincide con otras metodologías (como la indagación, planteamiento CTS o ABP) en problematizar, contextualizar e indagar, incluyendo el uso de fenómenos y la evaluación de pruebas como parte indispensable del proceso, lo hace dando gran valor al mundo de las ideas, no solo de los alumnos, sino también de la ciencia (escolar). Con ello brinda al alumnado la posibilidad de participar de una práctica epistémica suficientemente genuina pero no 
naíf, de forma que los estudiantes puedan ir construyendo y re-construyendo un modelo tentativo que se acerque cada vez más al MCE objeto de aprendizaje.

Para promover los procesos de modelización en el aula, varios autores han realizado propuestas de ciclos de modelización (por ejemplo, Halloun, 2016; Schwarz et al., 2009). En nuestra investigación utilizamos la propuesta de Garrido Espeja (2016) y Couso (2020), la cual, a diferencia del resto, separa las prácticas de modelización que se quieren promover en el alumnado de la secuencia didáctica que debería seguir el profesorado para conseguirlo (figura 1). El ciclo está compuesto por seis fases que orientan el diseño de Secuencias de Enseñanza y Aprendizaje (SEA), siendo la fase 1 especialmente útil para problematizar (a través de un fenómeno que «ancle» el conocimiento) y generar un interés para modelizar, y el resto de las fases como promotoras de distintas prácticas de modelización.

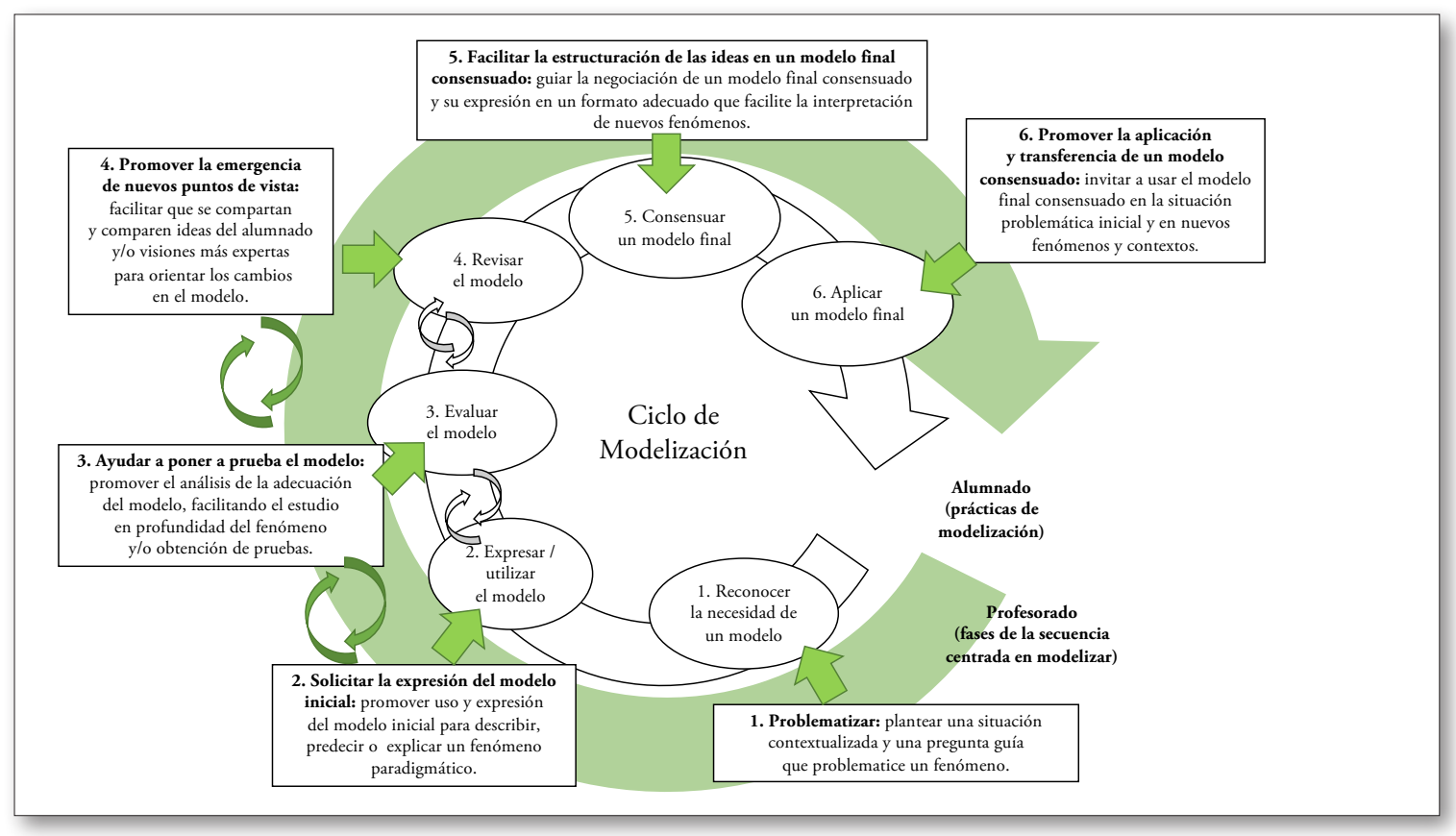

Fig. 1. Ciclo de modelización propuesto en Garrido Espeja (2016) y actualizado en Couso (2020).

Desde nuestra perspectiva, para conseguir formar a docentes competentes en la enseñanza basada en la modelización, son necesarios dos componentes. En primer lugar, es fundamental que los FD experimenten en primera persona los detalles y las características de este enfoque didáctico (MartínezChico et al., 2014; Davis, 2003), de manera que les permita reconocer en qué consiste la modelización y a la vez experimentar el reto que supone construir progresivamente los MCE objeto de enseñanza.

En segundo lugar, la metarreflexión didáctica es especialmente relevante en contextos de formación inicial, ya que permite empezar a introducir, de manera muy ligada a la propia vivencia como alumnos, el conocimiento pedagógico del contenido que los FD deben tener. En línea con otros autores (Martínez-Chico et al., 2014; Schwarz et al., 2009), entendemos la metarreflexión didáctica como la reflexión explícita con los FD de los aspectos didácticos, paso imprescindible para conseguir una transferencia adecuada del conocimiento didáctico. Ahora bien, esta reflexión explícita no debe hacerse de manera teórica, abstracta y aislada (lo que ha demostrado no tener éxito por quedar descontextualizada de la realidad del alumnado), sino que se debe conectar directamente con la propia experiencia vivida en el aula (Martínez-Chico et al., 2014). 
Por último, sabemos que la enseñanza y aprendizaje de las ciencias puede suponer ciertas tensiones (o dilemas) entre la autenticidad de la disciplina, la relevancia personal y la experiencia escolar vivida en el aula de ciencias (Kapon, Laherto y Levrini, 2018). Estas tensiones son multifacéticas y altamente contextuales, por lo que pueden variar enormemente de un contexto a otro. Estudios previos hacen una exploración de posibles tensiones en proyectos STEM (Kapon et al., 2018; Pérez-Torres, Couso y Márquez, 2021), aunque no concretamente sobre modelización. En este estudio pretendemos iniciar una exploración de tensiones que existen en relación con la modelización como enfoque didáctico, a partir de la visión que tienen de ella los propios participantes de una formación inicial docente centrada en modelizar para construir unos MCE clave, en dos contextos educativos muy distintos tanto a nivel académico como cultural.

\section{PREGUNTAS DE INVESTIGACIÓN}

En concreto, en esta investigación respondemos a las siguientes preguntas:

1. ¿Qué tipo de actividades formativas de la SEA que vivieron los FD valoran estos como útiles para la construcción de conocimiento?

2. ¿Qué tensiones didácticas emergen de las percepciones de los FD sobre la enseñanza de las ciencias centrada en la modelización?

\section{METODOLOGÍA}

El diseño de investigación se enmarca en una metodología cualitativa-interpretativa (Sandín, 2003), con la cual se busca conocer en profundidad las percepciones de los FD respecto al enfoque metodológico de enseñanza de las ciencias centrado en la modelización que han vivido. Mediante el estudio de caso se busca identificar características distintivas que nos permitan identificar ciertos rasgos presentes en estos dos contextos de formación inicial específicos. Integramos una visión cuantitativa de las representaciones y un análisis cualitativo de los datos.

\section{Contexto de la investigación y participantes}

Esta investigación se ha llevado a cabo en dos contextos educativos diferentes. En el primer contexto participaron 70 FD de primaria que cursaban la asignatura «Didáctica de las Ciencias Experimentales» durante el curso 2015-16, en su tercer año del grado de Educación Primaria en la Universidad Autónoma de Barcelona (UAB), Cataluña, España. En el segundo contexto participaron 22 FD de física de secundaria que cursaban la asignatura «Bases Físicas de los Seres Vivos y su Medio Ambiente» durante el curso 2016, en su segundo año de la carrera de Pedagogía en Física y Matemática en la Universidad de Santiago de Chile (USACH). Las edades de estos participantes fluctuaban entre los 19 y 24 años y predominó la presencia de sexo femenino en primaria y masculino en secundaria (figura 2).

Nos interesa contrastar estos dos grupos de FD para poner a prueba el valor del ciclo de modelización en dos contextos conocidos por las autoras con culturas formativas diferentes. Por una parte, la formación en ciencias de los FD de primaria en Cataluña, aunque escasa, se caracteriza por ser mayoritariamente competencial y centrada en la realización de actividades experimentales en el aula. Por el contrario, en Chile en la formación inicial predomina una visión de la enseñanza centrada en el conocimiento disciplinar y el lenguaje científico por encima del desarrollo de competencias y de prácticas científicas genuinas (Cofré et al., 2010). 


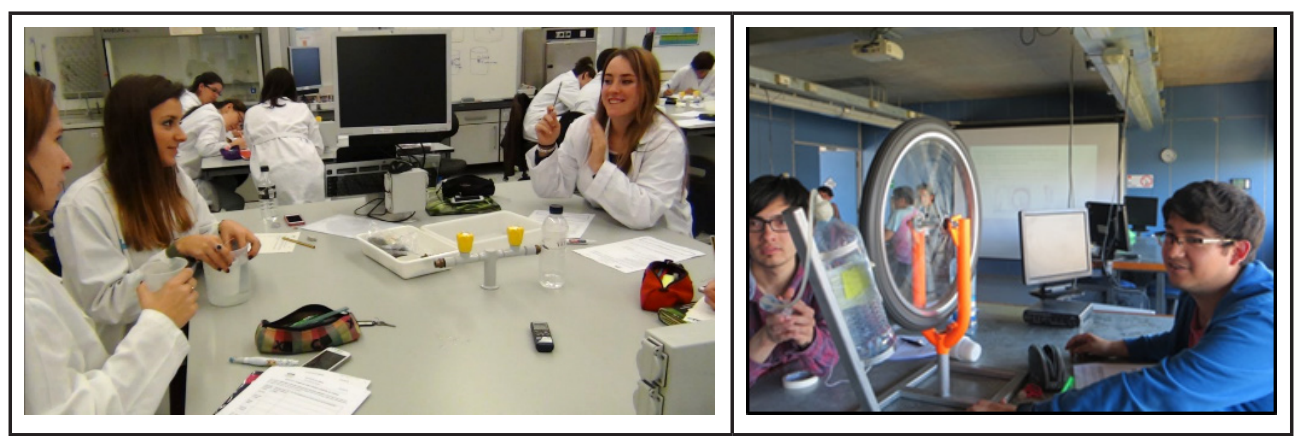

Fig. 2. A la izquierda imagen de un grupo de FD de primaria de Cataluña y a la derecha de FD de secundaria de Chile.

En ambos contextos diseñamos, implementamos y evaluamos, iterativamente y a lo largo de tres cursos, una SEA de formación docente análoga, usando el Ciclo de Modelización (figura 1) y planteando actividades similares (experimentales, simuladores virtuales, puestas en común, etc.). Sin embargo, la selección de los MCE de cada contexto fue distinta, considerando los requerimientos de los programas de las asignaturas de cada institución. Para el caso de primaria, los MCE eran cuatro: modelo de cambio geológico, de cambio químico, de interacciones newtonianas y de ser vivo. Las SEA se ejecutaron durante las 12 sesiones (de 2-4 horas) que duraba toda la asignatura. En el caso de secundaria, la SEA se ejecutó en 4 sesiones (de 4 horas) y se orientó a construir el MCE de energía. El resto del curso de secundaria fue impartido por otros docentes y no se pudo intervenir. La adecuación didáctica de las SEA y su utilidad para la construcción de conocimiento científico escolar que se promovieron en la implementación de las SEA se analizan en las tesis doctorales de las dos primeras autoras (Garrido Espeja, 2016; Soto, 2019).

\section{Recogida y análisis de datos}

Para identificar las percepciones de los FD respecto a la modelización, se implementó un cuestionario semiabierto en la última sesión de ambos cursos. El cuestionario se basó en un diseño propuesto por Couso y Garrido (2017) y adaptado en Soto (2019) que constó de tres partes. En la figura 3 se resumen las preguntas e información incluida en el cuestionario.

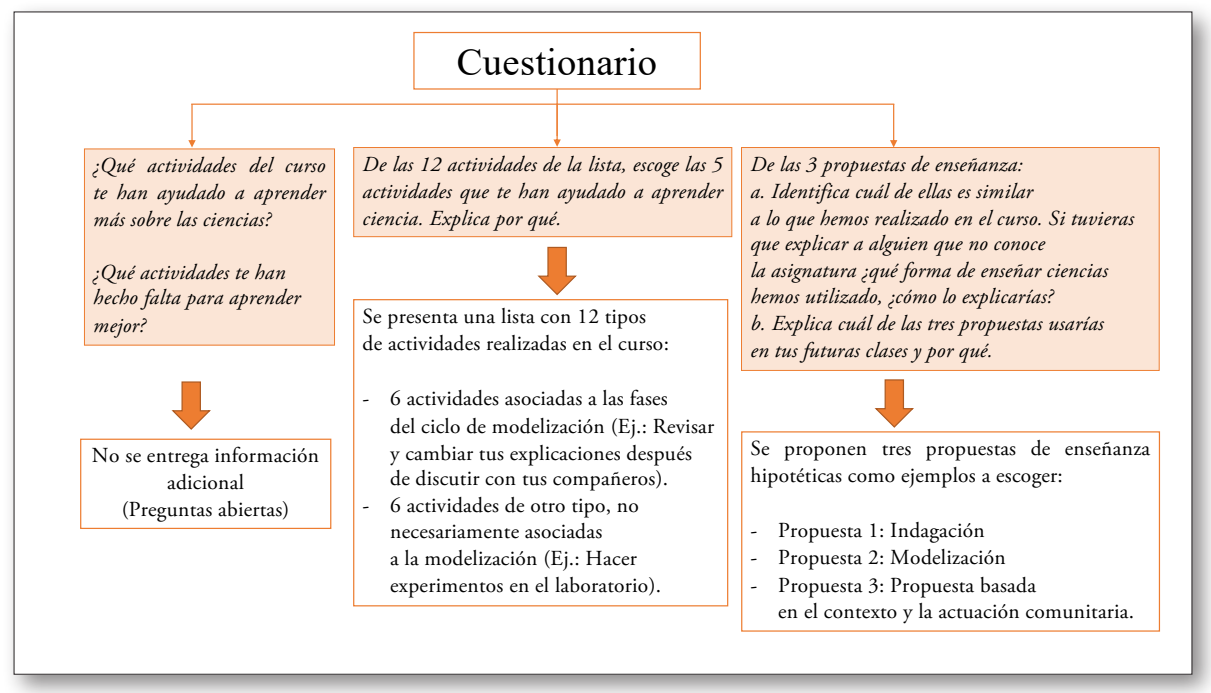

Fig. 3. Esquema del cuestionario. Incluye preguntas planteadas (recuadros naranjas) y la información proporcionada para responderlas (recuadros blancos). 
Las doce actividades que se presentan en la segunda parte del cuestionario fueron todas realizadas a lo largo de las SEA de formación docente, siendo seis de ellas correspondientes a las seis fases del ciclo de modelización y otras seis a actividades comunes en el aula de ciencias que no tenían necesariamente como objetivo modelizar (por ejemplo, «realizar experimentos»), a pesar de que estaban insertas dentro de una fase. Definimos como actividades todas aquellas «tareas concretas» que los estudiantes ejecutan dentro de alguna fase del ciclo de modelización. Las fases son el esquema o esqueleto que seguir, mientras que las actividades son la concreción de estas fases en un contexto y para un MCE determinado. La demanda de escoger las cinco actividades que más les sirvieron para aprender el MCE tenía como objetivo analizar si los FD priorizaban las actividades propias de la modelización u otras no propias de la modelización.

En la tercera parte del cuestionario nos interesaba saber si los FD eran capaces de identificar el enfoque didáctico usado durante la implementación de la formación y conocer si lo usarían en sus futuras clases. Para ello, se presentaron tres propuestas didácticas hipotéticas innovadoras y que estaban fundamentadas en enfoques didácticos que se habían presentado como interesantes a lo largo de la formación inicial (por ejemplo, la propuesta 3 consistía en resolver una problemática ambiental buscando información y generando una acción en la comunidad).

Los datos recogidos a partir de las respuestas de los FD se codificaron como P1, P2, ..., P70 (para FD de primaria) y $S 1, S 2, \ldots, S 22$ (para FD de secundaria).

Para dar respuesta a la pregunta de investigación 1 se analizaron, de manera separada para los dos contextos formativos, las respuestas de los FD a las partes 1 y 2 del cuestionario, identificando qué aspectos de los mencionados están relacionados con la modelización o con otro tipo de enfoques didácticos, y se compararon las diferencias y similitudes entre los dos grupos.

Para dar respuesta a la pregunta 2, se realizó un análisis transversal de las respuestas a todas las preguntas del cuestionario, identificando aquellas opiniones que mostraban relaciones de contradicción entre ellas por mostrar distintas interpretaciones o visiones en relación con un tema (por ejemplo, la experimentación), de manera similar a la realizada en Pérez-Torres et al. (2021). Por ejemplo, la contradicción que existe entre considerar la experimentación como una actividad interesante per se desde una perspectiva activista frente a considerarla un instrumento clave para la construcción de las propias versiones del MCE desde una visión modelizadora. De manera emergente, se fueron infiriendo de los datos diversas contradicciones, de las cuales se seleccionaron aquellas más relevantes didácticamente y más presentes en los grupos estudiados. En este caso, no nos interesa saber el porcentaje concreto de FD que se sitúan en cada tipo de contradicción, sino solo identificar el tipo de tensiones que pueden aparecer en relación con la práctica de modelización y si estas tienen relación con cada contexto estudiado o no. Para saber si el contexto educativo y sociocultural tiene alguna influencia, en cada una de las tensiones, se identificó si cada aspecto de la contradicción estaba igualmente presente o de manera más clara en un grupo en particular.

Esta organización e interpretación dio origen a cuatro tensiones clave, definidas como aspectos de la modelización sobre los que los FD tienen contradicciones.

La categorización de las respuestas fue inicialmente realizada por las investigadoras principales y posteriormente se puso a prueba a través de una triangulación con investigadoras externas al estudio. La confiabilidad fue mayor del $70 \%$ recomendada para el análisis cualitativo (Miles y Huberman, 1994) y las posibles diferencias de interpretación fueron solventadas a través de la discusión (Louca, Zacharia y Constantinou, 2011), hasta llegar a un consenso sobre la definición de las tensiones. 


\section{RESULTADOS Y DISCUSIÓN}

\section{Tipo de actividades valoradas por los futuros docentes}

$\mathrm{Al}$ analizar las respuestas abiertas de los FD (parte 1 del cuestionario), tanto los de primaria como los de secundaria mencionan dos tipos de actividades como útiles para aprender ciencias: aquellas propias de la modelización (mencionando explícitamente fases del ciclo de modelización) y actividades experimentales en general. Esta valorización se manifiesta aún más cuando los FD seleccionan las 5 actividades que más los ayudaron a aprender ciencias (parte 2 del cuestionario) y que detallamos a continuación.

\section{Actividades propias de la modelización}

Cuando los FD reflexionan en torno a actividades propias de la modelización, existen pocas diferencias entre los dos contextos educativos: todos los FD destacan que estas actividades les permiten desarrollar conocimiento de manera significativa, reconociendo explícitamente el valor de algunas fases del ciclo. En particular, la mayoría de ellos (entre el 45 y el $64 \%$; figura 4) consideran especialmente útiles las actividades asociadas a las fases de expresión inicial del modelo (fase 2), de aplicación (fase 6) y de consenso (fase 5). También valoran, aunque en menor medida, las fases de evaluar (fase 3) y revisar el modelo (fase 4).

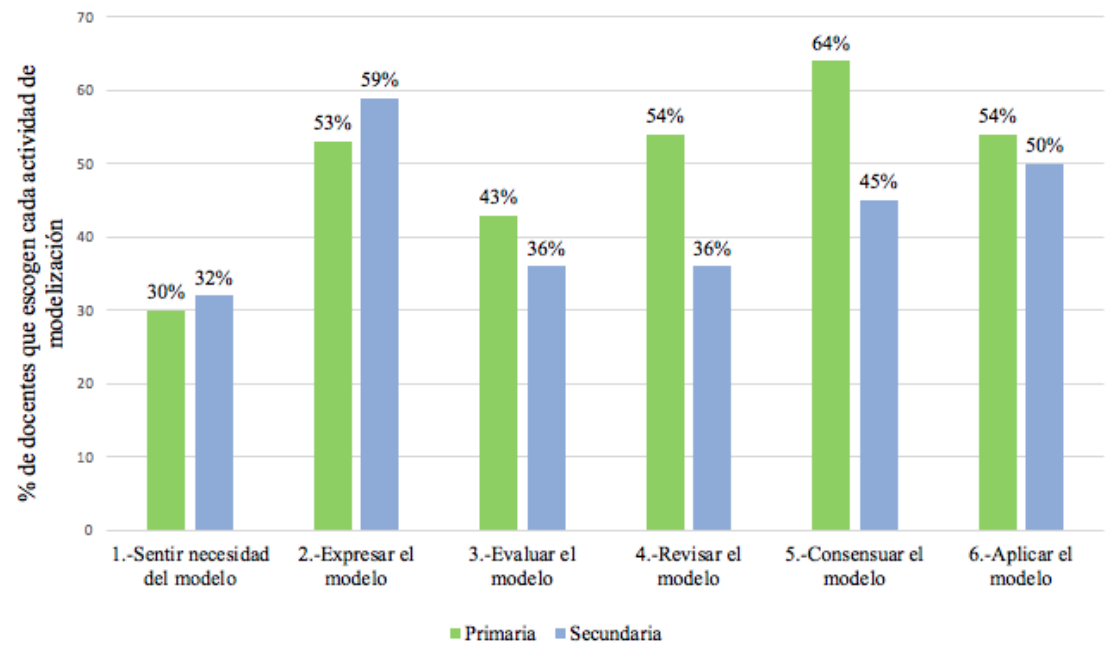

Fig. 4. Porcentaje de FD (primaria y secundaria) que escogen cada una de las actividades asociadas a las fases del Ciclo de Modelización como útiles para aprender ciencias.

En relación con la fase 2 (expresión inicial), ambos grupos (53\% de primaria y $59 \%$ de secundaria) señalan en sus justificaciones que las actividades asociadas a esta fase son clave para cambiar la manera de aprender ciencias a la que están acostumbrados y construir conocimiento de manera significativa, porque les permiten activar el pensamiento, conocer sus concepciones iniciales, motivarse para el aprendizaje y buscar respuestas:

P18 Porque es muy diferente como siempre me han enseñado: 'Es asi y punto'. Te hacen activar la imaginación y pensar qué pasará, te motivan a experimentar y encontrar respuestas. 
S7 Porque es bueno tener curiosidad y partir por los conceptos previos que tengo a la actividad y tener una referencia o consciencia de los conceptos que domino.

Ambos grupos de FD ( $54 \%$ de primaria y $50 \%$ de secundaria) también señalan que las actividades asociadas a la fase 6 (aplicación) fueron relevantes para llegar a aprender profundamente las ideas, considerando que si son capaces de usar el modelo en nuevos contextos quiere decir que lo han aprendido realmente:

P39 Este esfuerzo de aplicación es lo que hace que realmente aprenda.

S3 Es una buena forma de aplicar lo aprendido en un nuevo caso, ya que se analiza desde una nueva perspectiva y no nos cerramos solo a lo visto en clases.

Respecto a la fase 5 (consenso), también altamente valorada, existe mayor diferencia entre los dos grupos de FD (64\% de primaria y $45 \%$ de secundaria). En ambos casos, se destaca la importancia de la reflexión grupal, el diálogo y la generación de acuerdos con sus pares para reconstruir su conocimiento integrando ideas de los demás:

P63 Todo el mundo aporta ideas o conocimientos, se crea un debate, hipótesis, dudas... con consenso hacemos una conclusión compartida más valiosa.

S13 Se mejora la comprensión del conocimiento y se vuelve más valioso ya que existen aportes desde todos los puntos de vista.

La fase 3 (evaluación) y la fase 4 (revisión) fueron también valoradas por los FD, aunque en menor grado: $43 \%$ en primaria y $36 \%$ en secundaria (fase 3); y un $54 \%$ en primaria frente al $36 \%$ en secundaria (fase 4). Se resaltó la importancia de poder replantearse sus ideas sobre la base de los resultados de la experimentación, así como el uso de simulaciones y analogías para comprender ideas abstractas de los modelos:

P12 Hacer el experimento y encontrar una explicación [fase 3]

S11 Una de las actividades que más me ayudó, fue la de la caja de legumbres que representaban las moléculas, que con el calor se movian y al generar fuerzas se direccionaban [fase 4]

Las dos fases en las que se muestran mayores diferencias entre los dos grupos son las fases 4 y 5 , en ambos casos más valoradas por los FD de primaria que por los FD de secundaria. Este resultado lo podríamos atribuir a la mayor inseguridad respecto al contenido científico que tienen los FD de primaria. $\mathrm{Al}$ ser estas las fases en las que se revisan y estructuran las ideas científicas de manera conjunta y con la ayuda del docente, resulta natural que sean más valoradas por aquellos FD con menor formación científica previa.

La fase menos valorada por ambos grupos fue la fase 1 (sentir/reconocer la necesidad de un modelo), en la que el alumnado se enfrenta a un fenómeno contextualizado que aún no es capaz de explicar. En esta fase no se pretende que el alumnado se implique en una práctica de modelización explícita, ya que se trata de un diálogo oral en el que no necesariamente se expresa el modelo, y es más bien una fase "precursora» de la posterior práctica de modelización. En ese sentido, es comprensible que esta fase no se resalte por los FD que participaron como alumnos, aunque sí se trate de una fase relevante a nivel didáctico, ya que es clave para promover el interés inicial y la motivación de los estudiantes para querer construir un modelo a través de una pregunta que «enganche» (Jiménez-Liso, 2020) que los lleve a querer «saber más». 


\section{Actividades no propias de la modelización}

En cuanto a aquellas actividades no propias de la modelización, las más valoradas por todos los FD fueron aquellas asociadas a la experimentación en el laboratorio (86 \% en primaria y $55 \%$ en secundaria) y tener las ideas clave escritas al final de la secuencia (60 y $50 \%$, respectivamente) (figura 5).

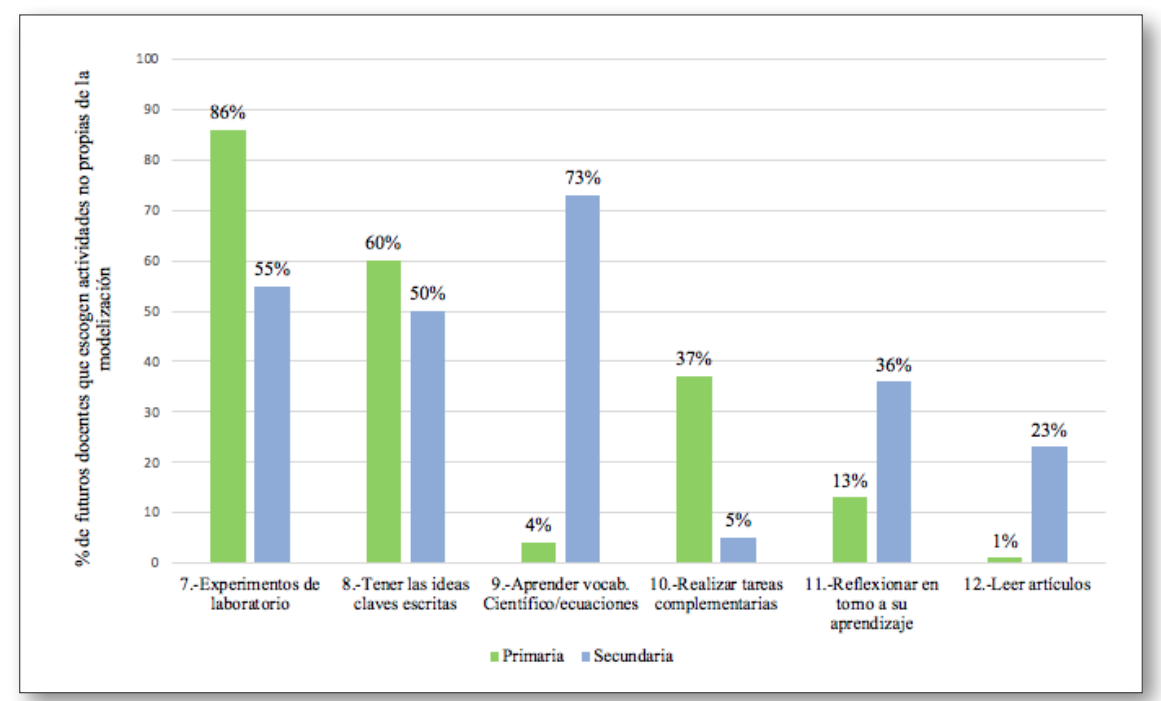

Fig. 5. Porcentaje de FD (primaria y secundaria) que escogen actividades no propias de la modelización.

En el caso de la experimentación (actividad que forma parte de la fase 3 del ciclo de modelización, pero que se presenta en el cuestionario como actividad puramente experimental/manipulativa), aunque ambos grupos la valoran, es especialmente relevante para los FD de primaria, para los que constituye la actividad formativa más importante, por encima de otras actividades de modelización.

Aun así, al analizar las respuestas abiertas de ambos grupos, se observa que son minoría los FD (también de primaria) que justifican su elección valorando la experimentación por sí misma y desconectada de un proceso de modelización (aquellos que se centran en que esta les permite «ver lo que pasa», o que es más efectiva que las clases teóricas, etc.). Al contrario, la mayoría de los FD valoran estas actividades experimentales desde una perspectiva que podríamos denominar modelizadora, propia de la ACE, al relacionar la experimentación con la construcción de modelos y la puesta a prueba de ideas, mencionando aspectos propios de la fase de evaluación (como la discusión de ideas o la comprobación de hipótesis), más que a experimentar per se:

P11 Trabajar en el laboratorio nos permite trabajar en pequeño grupo y favorece la interacción, el debate, poner en común argumentos e hipótesis.

Respecto a la actividad de tener las ideas clave escritas al final de la secuencia, se realizó dentro de la fase 5 de consenso y está altamente relacionada con esta fase. Al seleccionarla más que la fase 5 y después de analizar sus justificaciones, sin embargo, creemos que los FD dan más importancia al objetivo final de saber cuáles son las ideas más importantes que deben tener claras al final de un tema que al proceso de consensuarlas y, por lo tanto, valoran recibir las ideas científicas redactadas de forma ortodoxa por la docente, más que construirlas conjuntamente y con sus propias palabras: 
S2 Una vez obtenida la idea principal, tenerla bien redactada y con muchos detalles sirve para comparar y nuevamente trabajarla de manera más fina para un resultado final.

Con respecto al resto de las actividades, hay diferencias importantes en las preferencias de ambos contextos formativos. Un caso interesante es la gran diferencia detectada respecto a la actividad de aprender vocabulario científico y ecuaciones, con un $73 \%$ por parte de los FD de secundaria frente solamente a un $4 \%$ por los FD de primaria. Los FD de secundaria justifican su elección sobre la base de la creencia de que conocer el vocabulario científico correcto les permitirá comprender los fenómenos, tener un lenguaje compartido e inequívoco o no tener errores en los conceptos científicos (de manera que no los transferirán a sus futuros estudiantes):

S6 Conocer el vocabulario correcto cientificamente nos permite una mejor comprensión de los fenómenos físicos y homogeneización para tratar estos temas, lo que no deja espacio para los malentendidos lingüisticos que puedan influir en el aprendizaje.

Esta valoración se acerca a una visión sintáctica -ecuaciones- de abordar la ciencia que iría en contra de la visión de construcción de modelos propia de la ACE y de la epistemología semanticista. Atribuimos esta diferencia a la cultura educativa de procedencia de los PF de secundaria, formados en carreras científicas que, en general, se relacionan con mayor presión respecto a la «adecuación» científica y una preferencia por los aspectos teóricos (Cofré et al., 2010).

Esta gran diferencia entre los dos grupos también se observa en el resto de las actividades menos escogidas: la realización de tareas complementarias (más valorada en primaria), la reflexión en torno a su propio aprendizaje y la lectura de artículos (más valoradas en secundaria).

\section{Tensiones didácticas sobre modelización que emergen de las percepciones de los FD}

A partir de un análisis detallado y cruzado de las respuestas de los FD logramos identificar cuatro tensiones didácticas. Las primeras dos tensiones $\left(\begin{array}{lll}1 & \text { y }\end{array}\right)$ involucran visiones excluyentes sobre un tema, donde solo una de ellas es compatible con la enseñanza centrada en la modelización. En las siguientes dos tensiones (3 y 4), las dos visiones no son necesariamente excluyentes y pueden ser compatibles y convivir entre ellas a pesar de existir una cierta contradicción. En cualquier caso, todas estas suponen desafíos para la enseñanza y aprendizaje de modelos a través de la modelización y, por lo tanto, las consideramos potencialmente interesantes para discutir y tener en cuenta a la hora de plantear clases modelizadoras.

\section{Tensión 1. La experimentación como activismo vs. como instrumento para la construcción de modelos}

Esta tensión se manifiesta al analizar el valor que dan los FD a las actividades experimentales. Mientras que algunos las valoran per se, por el hecho de ver los fenómenos en primera persona o por hacer el aprendizaje más atractivo:

P32 Podía verlo con mis propios ojos.

Otros la valoran por su papel en el proceso de construcción de conocimiento, al poner a prueba sus ideas en contextos reales: 
S16 Es fundamental tener una representación del fenómeno para asi poder observar los comportamientos de los fenómenos y analizarlos en forma grupal para asi aclarar nuestras concepciones acerca del fenómeno.

En la literatura de enseñanza y aprendizaje de las ciencias esta tensión es muy relevante, al relacionarse la inclusión de experimentación per se en el aula con enseńanza innovadora. En nuestro ciclo de modelización consideramos la observación y experimentación de los fenómenos como parte integral y necesaria para hacer avanzar nuestras ideas sobre cómo es y sucede un fenómeno. Esto significa incluir actividades de indagación de tipo minds-on (cognitivas o que involucran mentalmente), diferentes de aquellas actividades prácticas centradas en la simple manipulación motivacional (experimentación vistosa sin sentido conceptual) (Ogborn, 2012) o en el seguimiento de una receta para la «verificación» de la teoría (experimentación demostrativa).

Esta tensión hace evidente la necesidad de hacer conscientes a los FD del tipo de trabajo práctico que queremos promover como docentes, relacionado con la puesta a prueba de las propias ideas y no con un activismo supuestamente motivacional.

\section{Tensión 2. El profesorado como único responsable de la estructuración del conocimiento vs. la comunidad como protagonista en la coconstrucción de ideas}

Los FD valoran las actividades de generar acuerdos y negociar significados consensuados con sus pares y el profesorado, es decir, actividades propias de la estructuración consensuada del conocimiento (fase 5).

P23 Es importante encontrar una respuesta con la que todos estemos de acuerdo y que todos podamos entender.

Sin embargo, son más los FD que solicitan en sus reflexiones la formalidad, por parte del profesorado, del contenido conceptual que aprender tal cual «dice la ciencia». Es decir, solicitan tener plasmada una expresión formal del modelo objeto de aprendizaje de manera ortodoxa, en forma de lista de ideas, esquemas, texto narrativo o en el caso de los FD de secundaria a través de ecuaciones y vocabulario científico específico.

P54 Tener bien redactadas las ideas me ayuda a tener presentes las ideas que hemos trabajado y me van muy bien a la hora de estudiar.

Esta tensión es muy relevante porque apela no solo a quién tiene la responsabilidad en la estructuración del conocimiento, sino también a qué visión de la naturaleza del conocimiento científico escolar tienen los FD: más centrada en los productos (teoría) de la ciencia escolar que en sus procesos dialógicos y sociales (Duschl y Grandy, 2008). Además, se relaciona con el hecho de que los FD no siempre son conscientes que en cada oportunidad educativa no aprendemos un conocimiento científico final, sino una "versión suficientemente adecuada» del modelo científico objeto de aprendizaje (Couso, 2015). Esto es esperable que se dé en ambos contextos formativos, aunque fue más mencionado en secundaria, posiblemente porque estos FD suelen tener experiencia en metodologías de aula en las que tienen un papel mucho más pasivo como receptores de la información.

Para lidiar con esta tensión en el aula de ciencias, consideramos esencial hacer explícita la descentralización del rol del docente como agente poseedor del conocimiento, lo que refleja mejor la naturaleza de la ciencia y el quehacer científico real. 


\section{Tensión 3. La importancia de construir MCE clave frente a la alta demanda temporal y cognitiva que implica}

Los FD son conscientes de la enorme implicación y esfuerzo cognitivo que supone construir conocimiento de manera conjunta en el aula a través de la modelización. En concreto, destacan la falta de tiempo para procesar las nuevas ideas del modelo que emergían en las sesiones, así como falta de momentos de descanso y calma para integrar y fortalecer su comprensión de estas:

\section{S7 El factor tiempo, en momentos trabajamos apurados y eso dificultó el aprendizaje.}

Esta percepción es comprensible, puesto que las sesiones en ambos cursos fueron muy intensas, tanto cognitivamente como respecto al número de actividades y contenido conceptual. Además, hay que tener en cuenta que los MCE planteados (por ejemplo, el modelo de interacción/flotación y el modelo de energía) son modelos especialmente abstractos y antiintuitivos.

En este sentido, además de diseñar formaciones donde se dé más tiempo al alumnado para construir y asentar sus ideas, creemos imprescindible reflexionar con los FD sobre las implicaciones de la naturaleza de los MCE, y entender que para llegar a construir y reconstruir ideas abstractas y complejas se requiere de tiempo. Es justo esta naturaleza abstracta, relacionada con su potencial tanto de aplicación como de desarrollo del pensamiento, la que hace necesario usar metodologías demandantes, como aquellas basadas en la modelización, para su aprendizaje. En cambio, otros contenidos científicos de corte declarativo (por ejemplo, las partes del sistema digestivo) no requerirán de un proceso tan exhaustivo.

\section{Tensión 4. La modelización como enfoque de gran valor didáctico frente a la inseguridad para promoverla como docente}

Por último, existe una tensión muy interesante en los FD entre reconocer el valor de la modelización que han vivido en primera persona y su falta de convicción, en algunas ocasiones, para usar este tipo de metodologías en sus futuras clases, al ser conscientes de la dificultad que supone llevarlas a la práctica como docentes. Así, por un lado, los FD valoran la modelización por su autenticidad y la posibilidad que les brinda para reformular sus concepciones erróneas, discutir con sus pares y llegar a sus propias conclusiones:

S12 Me gustó el hecho que fuera distinta a las demás clases, me hizo pensar [...]. Me parece buena metodología, ya que nos pide nuestras concepciones acerca del tema, para asi luego verificar si son correctas y si no lo son para corregir.

Sin embargo, algunos de ellos reconocen que enseñar a través de la modelización es un gran desafío para el que no se sienten del todo capacitados, sobre todo en el caso de primaria, probablemente por su percepción de desconocimiento del contenido científico:

P15 Es una propuesta eficaz pero que necesita mucha preparación y organización del maestro.

S14 No me siento preparado para hacer una clase tan didáctica, donde surgirian muchas dudas y no tengo herramientas para resolverlas, no me gusta que me pregunten y no sepa resolverlo.

Algunos FD también declaran que usarían la modelización, pero de forma conjunta con otras propuestas, como la de indagación (por la participación activa al experimentar) o con la propuesta de actuación comunitaria (por la contextualización real e implicación), mostrando un conocimiento amplio de la realidad del aula, que no se limita a un único enfoque didáctico. 
Para avanzar en este aspecto, se deberían promover prácticas en la formación inicial que empoderen a los FD a sentirse capaces de llevar a cabo estas metodologías modelizadoras y otras compatibles con sus futuros alumnos, y reflexionar con ellos en torno a cuándo es deseable (y cuándo no) hacer uso de la modelización en el aula.

\section{CONCLUSIONES, IMPLICACIONES Y LIMITACIONES}

Este estudio aporta detalles sobre la visión y el valor que le dan los FD de ciencias a una metodología didáctica basada en la modelización después de haberla vivido como alumnado, indicando qué aspectos de esta valoran especialmente. Además, planteamos cuatro tensiones didácticas en relación con la modelización como enfoque didáctico en el aula de ciencias.

En los contextos educativos estudiados, las actividades de modelización fueron altamente valoradas para la construcción de ideas científicas, lo que aporta a la literatura que aboga por incluir la modelización desde del aula primaria (Acher et al., 2007; Schwarz y Gwekwerere, 2007; Windschitl et al., 2008). En concreto, los resultados indican que los FD valoran especialmente aquellas actividades de expresión inicial de ideas, de consenso y de aplicación de las ideas finales del MCE. Es decir, desde un posicionamiento apropiadamente constructivista, entienden la importancia de explicitar su modelo inicial y ser capaz de transferir el modelo final aprendido.

Sin embargo, los FD también dan valor a otras tareas no necesariamente centradas en la modelización y que pueden indicar una visión del conocimiento científico (y escolar) inadecuada, dando importancia a la experimentación naif (como simplemente motivadora y de «aprender viendo») o al tener las ideas clave del MCE bien escritas al final de la unidad (desde una visión centrada en el profesor como agente de conocimiento). Esto nos muestra que, aunque experimentar en primera persona aquellas metodologías que se espera que apliquen más adelante como docentes es seguramente una condición esencial, tal y como sugiere la literatura (Martínez-Chico et al., 2014; Davis, 2003), puede no ser suficiente. De hecho, nuestros resultados apuntan a que es necesaria una reflexión más profunda con los FD no solo respecto a qué tipo de actividades les hacen aprender, sino sobre todo a qué es aquello que les hace aprender en cada actividad y qué valor otorgan a ese aprendizaje (por ejemplo, a una versión consensuada y adecuada, aunque no final, del modelo). En este sentido, a pesar de la buena valoración que hemos tenido en ambos grupos sobre la formación siguiendo el ciclo de modelización, sería necesario compartir explícitamente esta herramienta con los FD para ayudarlos a reconocer aspectos de la metodología que no valoran especialmente.

El análisis de las visiones de los FD nos muestra, como ya podíamos esperar, que estas a menudo contradicen la visión consensuada por la literatura en didáctica. El valor de nuestro trabajo, sin embargo, reside en identificar aquellas visiones que pueden dificultar la comprensión, valoración y práctica futura de la enseñanza de las ciencias centrada en modelos y modelización. Que las mismas tensiones aparezcan en contextos formativos y culturales muy distintos señala el gran reto que supone la enseñanza de las ciencias modelizadora para la construcción de MCE, lo que presenta dificultades análogas independientemente del grado de familiarización previa con un modelo de enseñanza y aprendizaje competencial, o del dominio del contenido conceptual.

La primera tensión muestra que hay dos maneras de interpretar la experimentación en el aula de ciencias: como innovación motivadora y alternativa a la clase "teórica» tradicional, visión ya reportada como habitual entre los docentes en formación (Appleton, 2005), o como herramienta para la reconstrucción de nuestras explicaciones o modelos (Duschl y Grandy, 2008). Para promover que los FD adquieran cierto criterio respecto a qué tipo de trabajo práctico es el más adecuado para aprender ciencias, creemos necesaria en la formación inicial una reflexión explícita en torno a la modelización y 
la función que tiene en ella la experimentación, la cual debe permitir conectar con la teoría, es decir, las ideas propias y las de la ciencia, para construir conocimiento (Izquierdo, 2005).

La segunda tensión se refiere al estatus y agencia del conocimiento científico escolar: por un lado, los FD valoran la posibilidad de ser capaces de consensuar el conocimiento por sí mismos, y por otro, sienten el peso de la responsabilidad de hacerlo y manifiestan preocupación por si sus conclusiones no son realmente científicas, requiriendo un rol docente como autoridad que aporta el conocimiento. Estos resultados reflejan la dificultad de pasar de una orientación didáctica mayoritariamente transmisiva de los productos de la ciencia, a la que están acostumbrados, a una visión de auténtica construcción del conocimiento (modelización) más parecida a los procesos propios de la ciencia (Duschl y Grandy, 2008) que implica más responsabilidad personal. Para lidiar con esta tensión, es imprescindible reflexionar sobre ella en los contextos de formación modelizadora, dejando clara la importancia de un equilibrio entre la necesaria descentralización del rol del docente (evitando presentar directamente el modelo final) y el total relativismo del contenido (no cualquier modelo es válido si estamos aprendiendo ciencia escolar). De manera paralela, será esencial gestionar como docentes este frágil equilibrio, por un lado, promoviendo que sea el alumnado el que extraiga conclusiones de manera consensuada con sus pares, y, por otro, guiando la estructuración de contenidos y facilitando maneras más "científicas» de expresar las ideas construidas, a través de listados de ideas claves, lecturas u otros. En el caso de la formación docente, además, nos deberemos asegurar de que los FD entiendan que: 1) esas ideas finales son una versión didácticamente adecuada para un cierto nivel educativo de aquello que la ciencia sabe del tema, y 2) sus futuros alumnos no parten de esas ideas, sino que tendrán que pasar por el mismo proceso que han pasado ellos de construcción y reconstrucción de ideas constante.

La tercera y cuarta tensión están muy relacionadas, y tienen que ver con el contraste entre el valor dado a la modelización como enfoque metodológico efectivo para construir conocimiento científico (MCE) y su elevado coste y dificultad, tanto al vivirla (por su alta demanda cognitiva y falta de tiempo para integrar ideas) como para llevarla al aula como docentes (por los requerimientos que implica a nivel de dominio del contenido científico y de diseño, así como el valor dado a otras metodologías activas que no priorizan la construcción de conocimiento).

Para superar estas tensiones, creemos que las formaciones iniciales deberían brindar a los FD más oportunidades de experimentar en primera persona propuestas de modelización de diferentes MCE, posibilitando que aprecien que, a pesar de la aparente gran cantidad de conocimiento científico que hay que enseñar y aprender, en realidad este se puede reducir a través de una transposición didáctica a unas pocas ideas clave o MCE que se van construyendo a lo largo de toda la escolaridad. Para empoderar a los FD, proponemos ofrecerles buenos ejemplos de secuencias didácticas basadas en la modelización y que las tuvieran que adaptar e implementar en contextos de aula concretos (en sus prácticas, por ejemplo), en vez de hacerles diseñar de cero secuencias de este tipo, tarea que no es fácil para un profesor novel. Por último, consideramos que se debería compartir con los FD explícitamente la importancia de saber escoger la metodología didáctica adecuada (cómo enseñar) en función del contenido científico que se quiere construir (qué enseñar) (Couso, 2015; Izquierdo, 2005). El objetivo es que entiendan que participar en metodologías basadas en la modelización y plantearlas es un proceso costoso pero recomendable (y hasta imprescindible) para promover la construcción de las ideas centrales de la ciencia (los MCE) y que invertir tiempo en construirlas resulta beneficioso a largo plazo. Otras investigaciones han encontrado que los FD experimentan emociones positivas y negativas con las prácticas científicas de modelización, y que reconocen aprender modelos con estas (Jiménez-Liso et al., 2019).

Las tensiones presentadas no son fácilmente superables únicamente durante la formación inicial. Algunas son tensiones con las que convivirán nuestros FD durante toda su vida profesional. Sin embargo, consideramos de gran valor que estas emerjan explícitamente en la formación inicial, dotando de cierta madurez y criterio a su mirada profesional. En este sentido, creemos recomendable realizar 
formaciones iniciales equivalentes a la que hemos analizado, con el valor añadido que supone, precisamente, hacer emerger estas complejas cuestiones de forma explícita (Martínez-Chico et al., 2014; Schwarz et al., 2009). En especial, proponemos realizar una reflexión en la que se reconozca la importancia de "pensar lento» y "pensar tanto» (necesario para modelizar) en el aula de ciencias y el valor de invertir esfuerzos como docentes para llevar la modelización al aula (Halloun, 2016).

Este estudio tiene ciertas limitaciones metodológicas. En primer lugar, y a pesar de formar parte del mismo equipo de investigación y haber alineado tanto los diseños como la implementación con respecto a la modelización, existen diferencias contextuales insalvables, tanto entre las dos formadoras como entre las dos culturas formativas de referencia. A pesar de que la muestra no nos permite identificar diferencias significativas, creemos en el valor que aporta el ejercicio de ver en qué se parecen y en qué no los rasgos de valoración de una propuesta didáctica, en este caso de modelización, en diferentes contextos. Sería interesante ampliar el número de FD y contextos para ver si las tensiones identificadas también se dan en otros contextos o si aparecen otras. Además, sería recomendable explorar más estas tensiones, en diseños didácticos futuros, por ejemplo, o en su adaptación de estos materiales al aula. Por último, coincidimos con otros autores (Martínez-Chico et al., 2014) en que sería interesante ampliar la metarreflexión didáctica realizada con los FD durante la formación, y no solo al final de esta, favoreciendo una cultura reflexiva en el día a día de los participantes (por ejemplo, con un diario de sesiones).

\section{AGRADECIMIENTO}

Esta investigación ha sido financiada por el Ministerio de Ciencia, Innovación y Universidades (PGC2018-096581-B-C21) y llevada a cabo dentro del grupo de investigación ACELEC (2017SGR1399)

\section{REFERENCIAS BIBLIOGRÁFICAS}

Acher, A., Arcà, M. y Sanmartí, N. (2007). Modelling as a Teacher Learning Process for Understanding Materials: A Case Study in Primary Education. Science Education, 91(1), 398-418. https://doi.org/10.1002/sce.20196

Adúriz-Bravo, A. (2012). A «Semantic» View of Scientific Models for Science Education. Science \& Education, 22(7), 1593-1611.

https://doi.org/10.1007/s11191-011-9431-7

Appleton, K. (2005). Elementary science teacher education: International perspectives. Mahwah, NJ: Erlbaum.

Cofré, H., Camacho, J., Galaz, A., Jiménez, J., Santibáńez, D. y Vergara, C. (2010). La educación científica en Chile: debilidades de la enseñanza y futuros desafíos de la educación de profesores de ciencia. Estudios Pedagógicos (Valdivia), 36(2), 279-293.

Couso, D. (2015). La clau de tot plegat: la importància de "què» ensenyar a l'aula de ciències. Ciències: Revista Del Professorat de Ciències de Primària I Secundària, 0(29), 29-36. https://doi.org/10.5565/rev/ciencies.56

Couso, D. (2020). Aprender ciencia escolar implica construir modelos cada vez más sofisticados de los fenómenos del mundo. En D. Couso, M. R. Jiménez-Liso, C. Refojo, J. A. Sacristán (Coords.), Enseñando Ciencia con Ciencia. FECYT \& Fundación Lilly. Madrid: Penguin Random House. 
Couso, D. y Garrido, A. (2017). Models and modelling in elementary school pre-service teacher education: Why we need both. En K. Hahl, K. Juuti, J. Lampiselkä, A. Uitto, J. Lavonen (Eds.), Cognitive and Affective Aspects in Science Education Research (pp. 263-280). Suiza: Springer.

Davis, K. S. (2003). "Change is hard»: What science teachers are telling us about reform and teacher learning of innovative practices. Science Education, 87(1), 3-30. https://doi.org/10.1002/sce.10037

Duschl, R. A. y Grandy, R. E. (2008). Teaching Scientific Inquiry: Recommendations for Research and Implementation. Rotterdam, Países Bajos: Sense Publishers.

Garrido Espeja, A. (2016). Modelització i models en la formació inicial de mestres de primària des de la perspectiva de la pràctica cientifica. Barcelona: Universitat Autònoma de Barcelona. https://ddd.uab. cat/pub/tesis/2016/hdl_10803_399837/age1de1.pdf

Gilbert, J. K. y Justi, R. (2016). Models of modelling. En Modelling-based teaching in science education (pp. 17-40). Cham: Springer.

Halloun, I. (2016). Mediated Modeling in Science Education. Science \& Education, 16(6-7), 1-32. https://doi.org/10.1007/s11191-006-9004-3

Izquierdo, M. (2005). Hacia una Teoría de los Contenidos Escolares. Enseñanza de las Ciencias, 23(1), 111-122.

Izquierdo, M. (2007). Enseñar ciencias, una nueva ciencia. Enseñanza de las Ciencias Sociales, 6, 125138.

Izquierdo, M., Sanmartí, N. y Espinet, M. (1999). Fundamentación y diseño de las prácticas escolares de ciencias experimentales. Enseñanza de las Ciencias. Revista de Investigación y Experiencias Didácticas, 45-59.

Jiménez-Aleixandre, M. P. (2010). 10 ideas clave. Competencias en argumentación y uso de pruebas. Barcelona: GRAO.

Jiménez-Liso, M. R. (2020). Aprender ciencia escolar implica aprender a buscar pruebas para construir conocimiento (indagación). En D. Couso, M. R. Jiménez-Liso, C. Refojo, J. A. Sacristán (Coords.), Enseñando Ciencia con Ciencia. FECYT \& Fundación Lilly. Madrid: Penguin Random House.

Jiménez-Liso, M. R., Martínez-Chico, M., Avraamidou, L. y Lucio-Villegas, R. (2019). Scientific practices in teacher education: the interplay of sense, sensors, and emotions. Research in Science \& Technological Education, 39, 1-24. https://doi.org/10.1080/02635143.2019.1647158

Jiménez-Tenorio, N., Aragón-Nuñez, L., Blanco-López, A. y Oliva, J. M. (2016). Comprensión acerca de la naturaleza de los modelos por parte de profesorado de ciencias de secundaria en formación inicial. Campo Abierto, 35(1), 121-132.

Kapon, S., Laherto, A. y Levrini, O. (2018). Disciplinary authenticity and personal relevance in school science. Science Education, 102(5), 1077-1106. https://doi.org/10.1002/sce.21458

Lehrer, R. y Schauble, L. (2012). Seeding evolutionary thinking by engaging children in modeling its foundations. Science Education, 96(4), 701-724.

https://doi.org/10.1002/sce.20475

Louca, L. T., Zacharia, Z. C. y Constantinou, C. P. (2011). In Quest of productive modeling-based learning discourse in elementary school science. Journal of Research in Science Teaching, 48(8), 919951.

Márquez, C. y Artés, M. (2016). Propuesta de análisis de representaciones sobre el modelo cambio geológico del alumnado del grado de educación primaria. Enseñanza de Las Ciencias de La Tierra, 24(2), 169-181. 
Martínez-Chico, M., Jiménez-liso, M. R. y López-Gay, R. (2014). La indagación en las propuestas de formación inicial de maestros: análisis de entrevistas a los formadores de Didáctica de las Ciencias Experimentales. Enseñanza de las Ciencias, 32(3), 591-608. http://dx.doi.org/10.5565/rev/ensciencias. 1376

Miles, M. B. y Huberman, A. M. (1994). Qualitative data analysis: An Expanded Sourcebook (2.a ed.). Thousand Oaks: Sage.

Ogborn, J. (2012). Curriculum Development in Physics: Not Quite So Fast! Scientia in Educatione, 3(2), 3-15.

Oh, P. S. y Oh, S. J. (2011). What teachers of science need to know about models: An overview. International Journal of Science Education, 33(8), 1109-1130. https://doi.org/10.1080/09500693.2010.502191

Oliva, J. M. (2019). Distintas acepciones para la idea de modelización en la enseñanza de las ciencias. Enseñanza de las Ciencias: Revista de Investigación y Experiencias didácticas, 37(2), 5-24. https://doi.org/10.5565/rev/ensciencias.2648

Osborne, J. (2014). Teaching Scientific Practices: Meeting the Challenge of Change. Journal of Science Teacher Education, 25(2), 177-196.

https://doi.org/10.1007/s10972-014-9384-1

Pérez-Torres M., Couso D. y Márquez C. (2021) ¿Cómo diseñar un buen proyecto STEM? Identificación de tensiones en la co-construcción de una rúbrica para su mejora. Revista Eureka sobre Enseñanza y Divulgación de las Ciencias, 18(1), 1301.

https://doi.org/10.25267/Rev_Eureka_ensen_divulg_cienc.2021.v18.i1.1301

Sandín, M. (2003). Qualitative research in education. Barcelona, España: McGraw Hill.

Schwarz, C. V. y Gwekwerere, Y. N. (2007). Using a Guided Inquiry and Modeling Instructional Framework (EIMA) to Support Preservice K-8 Science Teaching. Science Education, 91(1), 158-186. https://doi.org/10.1002/sce.20177

Schwarz, C. V., Reiser, B. J., Davis, E. a., Kenyon, L., Achér, A., Fortus, D., ... Krajcik, J. (2009). Developing a learning progression for scientific modeling: Making scientific modeling accessible and meaningful for learners. Journal of Research in Science Teaching, 46(6), 632-654. https://doi.org/10.1002/tea.20311

Soto, M. (2019). Influencia de una propuesta formativa centrada en la modelización en la evolución del modelo cientifico escolar de energía en futuros docentes de física y matemática. Barcelona: Universitat Autònoma de Barcelona. https://ddd.uab.cat/pub/tesis/2019/hdl_10803_667161/mbsa1de1.pdf

Soto, M., Couso, D., López, V. y Hernández, M. I. (2017). Promoviendo la apropiación del modelo de energía en estudiantes de $4^{\circ}$ de ESO a través del diseño didáctico. Ápice. Revista de Educación Científica, 1(1), 90-106.

https://doi.org/10.17979/arec.2017.1.1.2003

Tena, E., Garrido, A. y López, N. (2018). ¿Cómo migran los pájaros? El uso de una noticia para la construcción de conocimientos científicos y matemáticos conectados en Primaria. UNO (Especial).

Windschitl, M., Thompson, J. y Braaten, M. (2008). How Novice Science Teachers Appropriate Epistemic Discourses Around Model-Based Inquiry for Use in Classrooms. Cognition and Instruction, 26(3), 310-378.

https://doi.org/10.1080/07370000802177193 


\title{
Pre-service science teacher education: possible contributions and tensions on modeling
}

\author{
Anna Garrido Espeja \\ Universidad Autónoma de Barcelona, Facultad de Educación, Barcelona, España \\ agarridoespeja@gmail.com \\ Macarena Soto Alvarado \\ Pontificia Universidad Católica de Chile, Facultad de Educación, Santiago, Chile \\ macarena.soto@uc.cl \\ Digna Couso Lagarón \\ Universidad Autónoma de Barcelona, Facultad de Educación, Barcelona, España \\ digna.couso@uab.cat
}

Modeling has been reported in science education as a relevant and useful scientific practice that allows students to build scientific knowledge, in particular school scientific models (Izquierdo et al., 1999; Oliva, 2019), improve their visions about the nature of science and achieve better results in lower achievers (Halloun, 2016). Despite these benefits, evidence shows that its use in school contexts is scarce (Schwarz \& Gwekwerere, 2007). For this reason, it is necessary that pre-service teacher training provide future teachers with the necessary tools and knowledge to rethink their pedagogical content knowledge and to learn innovative and coherent methodologies for their teaching practice, such as modeling-based teaching.

In our work, we designed and implemented two modeling-based teaching and learning sequences in pre-service teacher education courses in two different cultural contexts: Chile and Catalonia, Spain. In particular, the sequences were designed by following the modeling cycle proposed by Garrido Espeja (2016) and Couso (2020) and implemented by the researchers. By participating in these sequences, pre-service teachers experimented firsthand (as students) with modeling-based instruction to learn school scientific models.

The first aim of this research was to find out the perceptions of the future teachers in the two educational contexts, about modeling as a teaching methodology. The second aim was to identify possible tensions that may emerge from their visions about modeling-based instruction. Using a semi-open questionnaire, we analyzed the opinion of 70 future primary school teachers (in Catalonia) and 22 future secondary school teachers (in Chile) about the course in which they had just participated.

Results show that both groups value modeling as a very useful practice in which to participate for learning science and models, and both groups concur in particularly valuing the teaching phases that promote the initial expression of the model, the structuration of ideas in a consensus model and the transference and application of the model. However, they also highlight other aspects that are not specific to modeling but that they find valuable for learning science, which gives us ideas of how the implementation of a sequence that follows the modeling cycle can be approached. Certain variations between the two groups were also identified, which were discussed in terms of their cultural differences. We also identified four didactic tensions that should be taken into account when planning initial teacher education courses for science teachers: (1) experimentation as activism vs. experimentation as an instrument for the construction of models; (2) teachers as the only ones responsible for structuring knowledge vs. all the participants in the community as the responsible for the co-construction of ideas; (3) the importance of building key school scientific models vs. the high temporal and cognitive demand that this process implies; and (4) modeling as an approach of great educational value vs. the insecurity as a teacher to promote modeling. Finally, based on all the results, we discuss possible teaching and learning implications when teaching through modeling, we inform of methodological limitations of the study, and we suggest further research in this direction. 
